\title{
The ChemScreen project to design a pragmatic alternative approach to predict reproductive toxicity of chemicals
}

\author{
Bart van der Burga,*, Eva Bay Wedebye ${ }^{\mathrm{b}}$, Daniel R. Dietrich ${ }^{\mathrm{c}}$, Joanna Jaworska ${ }^{\mathrm{d}}$, \\ Inge Mangelsdorf ${ }^{e}$, Eduard Paune ${ }^{\mathrm{f}}$, Michael Schwarz ${ }^{\mathrm{g}}$, Aldert H. Piersma ${ }^{\mathrm{h}, \mathrm{i}}$, \\ E. Dinant Kroese ${ }^{\mathrm{j}}$
}



\begin{abstract}
A B S T R A C T
There is a great need for rapid testing strategies for reproductive toxicity testing, avoiding animal use. The EU Framework program 7 project ChemScreen aimed to fill this gap in a pragmatic manner preferably using validated existing tools and place them in an innovative alternative testing strategy. In our approach we combined knowledge on critical processes affected by reproductive toxicants with knowledge on the mechanistic basis of such effects. We used in silico methods for prescreening chemicals for relevant toxic effects aiming at reduced testing needs. For those chemicals that need testing we have set up an in vitro screening panel that includes mechanistic high throughput methods and lower throughput assays that measure more integrative endpoints. In silico pharmacokinetic modules were developed for rapid exposure predictions via diverse exposure routes. These modules to match in vitro and in vivo exposure levels greatly improved predictivity of the in vitro tests. As a further step, we have generated examples how to predict reproductive toxicity of chemicals using available data. We have executed formal validations of panel constituents and also used more innovative manners to validate the test panel using mechanistic approaches. We are actively engaged in promoting regulatory acceptance of the tools developed as an essential step towards practical application, including case studies for read-across purposes. With this approach, a significant saving in animal use and associated costs seems very feasible.
\end{abstract}

\section{Introduction}

The complexity of the system of risk assessment of chemicals does not allow rapid evaluation of basic toxicity profiles of chemicals. Because of this, the toxicological properties of most industrial chemicals that are in common use are largely unknown [1]. New legislation such as REACH aims to end this unacceptable situation by modernizing and streamlining chemical risk assessment [2].

\footnotetext{
* Corresponding author. Tel.: +31 20 4350750; fax: +31 204350757 .

E-mail address: bart.van.der.burg@bds.nl (B. van der Burg).
}

However, this approach is unlikely to be successful without incorporating alternative, integrated testing strategies in which costly and time consuming animal tests are replaced to a large extent by rapid and cost-effective alternative testing methods. An integrated testing strategy (ITS) is a method to combine test results from different sources, including non-test information (existing data, in silico extrapolations from existing data or modelling) to give a combined test result [3] Inclusion of in silico and in vitro test results and pre-existing data in principle allows more rapid evaluation of toxicological properties of chemicals. Such methods are particularly needed for reproductive toxicity testing of chemicals. Reproductive toxicity assessment is important for both man and 
the environment and uses relatively large numbers of experimental animals and time- and resource intensive testing procedures $[4,5]$. Unfortunately, there are very few if any alternative methods that are able to cover this complexity. The ChemScreen project aimed to fill this gap and select suitable tests and place them in a more general alternative testing strategy.

Because of the complexity of the process of mammalian reproduction intact organisms are often regarded to be essential in assessing reproductive toxicity of compounds. Even then, it has been shown that large species differences exist and interspecies extrapolation of developmental toxicity typically usually is not much higher than $60 \%$ between single species when using apical endpoints in animals [6]. Nevertheless, results from the Framework program (FP)6 program ReProTect very clearly showed that an in vitro test battery covering only part of the reproductive cycle processes can provide very promising results with respect to reproductive toxicity testing [7]. It has been argued that the use of in vitro tests that assess mechanisms of toxicity may form the basis for a new paradigm in toxicity testing and could also improve the possibility to extrapolate between species since pathways of toxicity share many similarities between different species $[8,9]$. This mechanism-based approach of toxicity testing was one of the cornerstones of the ChemScreen program. In 2005 we already developed a panel of mechanism-based CALUX assays to assess hormonal activity of compounds [10], a panel which has shown to be highly predictive for such activities in experimental animals [11,12]. Some of these mechanistic assays also formed a part of the promising battery of tests used in the ReProTect [7].

With this in mind the ChemScreen project has been designed, aiming at further simplification of the screening battery by increased use of preferably high throughput mechanistic screening assays [13]. We aimed to generate a rapid screening system, that is relatively simple, cost-effective, and can be implemented preferably within the tight time schedule of the REACH program. The tool should be flexible and adaptable for applications beyond this specific program, such as for safe design purposes, or prioritization. To fill the gap of suitable alternative methods for reproductive toxicity testing we used a novel high throughput approach combining in silico/in vitro methods. In this approach we combined knowledge of critical processes affected by reproductive toxicants with knowledge on the mechanistic basis of such effects. To reach our goals the following objectives were formulated, corresponding to the major ChemScreen work packages (WPs; see Fig. 1):

1. Establish in silico prescreening methods prioritizing in vitro toxicity testing.

2. Establish databases and in silico methods to identify potential reproductive toxicants.

3. Establishment of sensitive parameters and medium throughput in vitro assays.

4. Establish a high throughput mechanistic pathway screen for reproductive toxicants.

5. Establish methods to predict in vivo reprotoxicity.

Furthermore ample attention was paid to validation and application of the test methods developed. The ChemScreen project started at January 1,2010 . The program aimed to collaboratively generate an innovative testing strategy combining unique expertise of the participants. To attain this level of interaction, frequent meetings, and workshops were held, and a high profile Scientific Advisory Board was installed to help guide this process. In the international advisory board major stakeholders (JRC/ECVAM, OECD, US EPA, Industry, ECETOC) were represented. Beginning 2010 the ChemScreen project entered an important transatlantic

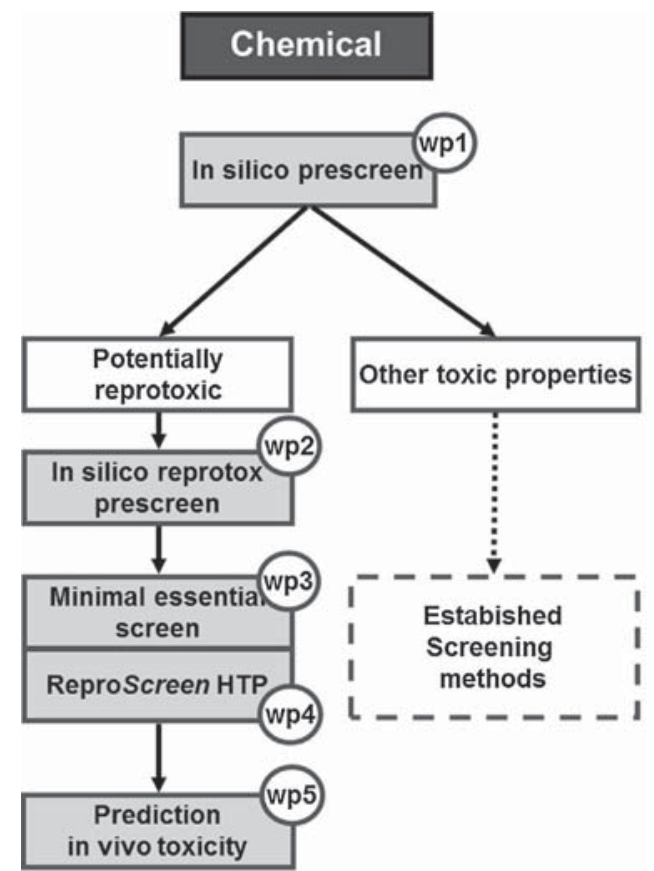

No testing/dedicated in vivo testing

Fig. 1. Graphical representation of ChemScreen's major components. The dotted lines indicate areas where alternative methods are relatively well developed or under construction in various (FP) programs in which partners participate. All workpackages (WPs) are indicated, except WP6 (ICT tools), WP7 (dissemination) and WP8 (management).

collaboration with the US EPA National Center for Computational Toxicology (NCCT), and the Texas Indiana Virtual Star Center (TIVSC). Agreements on scientific collaboration, data- and chemical sharing were established. Here we will present a summary of objectives and discuss obtained results within the work packages, and the overall picture that emerges from this. Specific materials and methodologies can be found in the referenced individual papers within this issue.

\section{Results and discussion}

2.1. In silico prescreening methods prioritizing in vitro toxicity testing

A crucial aspect of the REACH program is prioritization [2]. With, as it now turns out, about 50,000 chemicals needing an update of toxicological information within a decade, the potential amount of safety testing is enormous, which requires that chemicals of highest concern are identified for prioritized testing. An elaborate quantitative structure-activity relationship (QSAR) module aimed to allow prediction of the "leading health effect" as requested in REACH, avoiding unnecessary testing of compounds for which the leading health effect has been established. For example, compounds with mutagenic properties will not be evaluated for reproductive toxicity since further information on its potential carcinogenicity is of higher priority when considering leading health effects. Therefore, in this part of the project we concentrated on the establishment and selection of in silico prescreening methods to categorize chemicals, using QSARs. This was done for major classes of toxicity prioritized in REACH (carcinogenesis, mutagenesis and reproductive (CMR)) toxicity [2]. Positive predictions for carcinogenicity, 


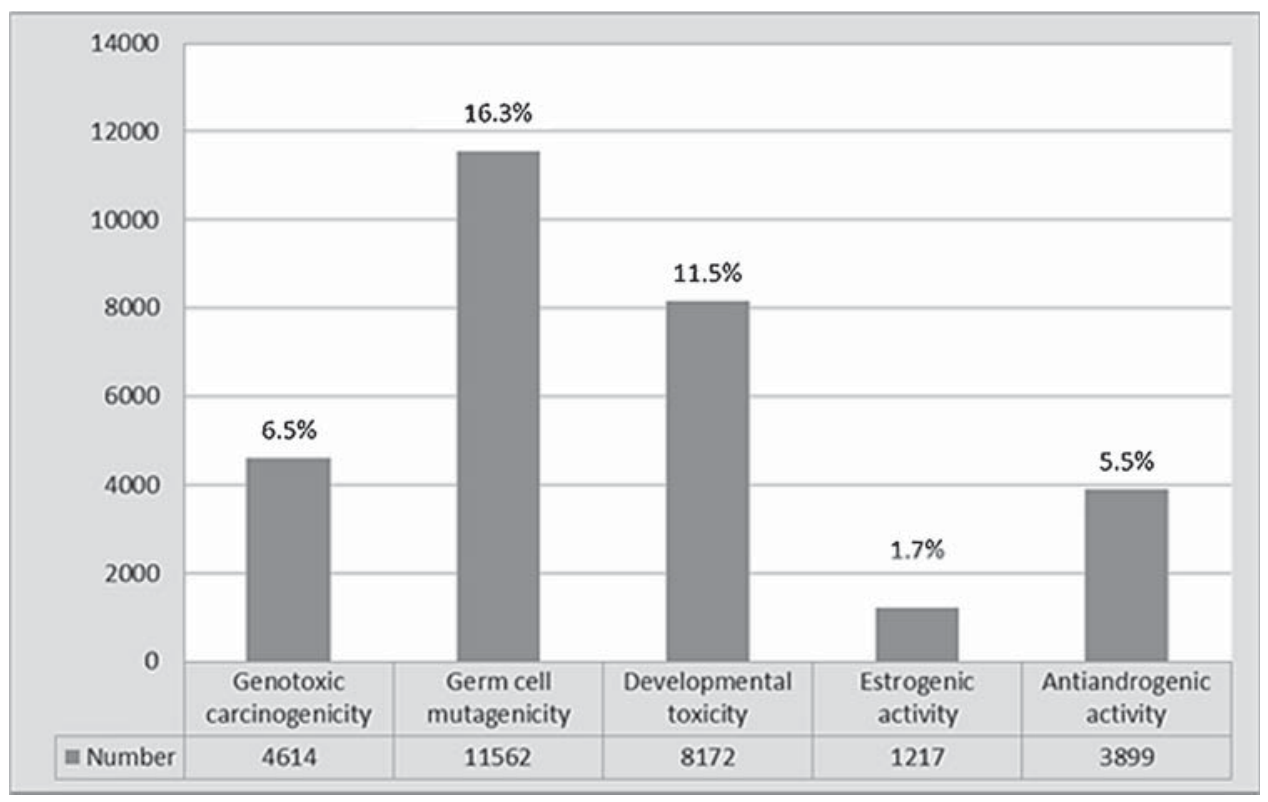

Fig. 2. Predictions using QSAR analysis using pre-screening tools for human health effects applied to over 70,000 REACH pre-registration chemicals [Wedebye et al., 2015].

mutagenicity and reproductive toxicity can be applied to avoid unnecessary animal testing of compounds. As no testing for reproductive effects should be performed in REACH on known genotoxic carcinogens or germ cell mutagens with appropriate risk management measures implemented, as stated in the REACH annexes VIII-X, predictions for genotoxic carcinogens and germ cell mutagens can furthermore be applied to avoid testing for reproductive toxicity. To make the QSAR screening as comprehensive as possible it was based on as many as possible of the 143,835 chemicals, which were pre-registered before December 2008. The predictions were based on structure information generated by the Computational Toxicology Group within the Joint Research Center (JRC) for 80,413 pre-registered substances (PRS), of which 70,983 chemicals were suitable for generation of QSAR predictions in the applied QSAR software [14]. Results are shown in Fig. 2 show that more than $25 \%$ of the chemicals have structural alerts of genotoxic carcinogens and germ cell mutagens, which will exclude them for being tested for reproductive toxicity. As part of the in vitro prescreen, it was also envisaged to develop an exposure module to identify chemicals that will have no or a negligible systemic availability (and anticipating that gonads and embryos will not be exposed) after exposure via different routes. To that effect, literature searches have been performed to investigate on one hand whether specific chemical properties can be identified to predict internal exposure after exposure via the oral, respiratory and dermal routes, and on the other hand which (publicly available) in silico tools exist leading to reliable prediction of negligible internal exposure. Based on the current knowledge, no cut-offs based on physicochemical properties could be identified for the three main exposure routes below or above which internal exposure is negligible [Buist and Verweij, unpublished results]. REACH does provide physicochemical criteria to waive toxicity testing via the respiratory route, but these criteria cannot waive testing via other routes of exposure. Therefore, it was concluded that inclusion of these criteria in a module to predict internal exposure aimed at excluding reproductive toxicity testing based on physicochemical criteria is not a viable option. Instead, the pharmacokinetic models will now be used exclusively in extrapolation of in vitro to in vivo data.

\subsection{Establish databases and in silico methods to identify potential reproductive toxicants}

A next step in our strategy was to further expand the possibilities of identifying potential reproductive toxicants without further testing. Databases were expanded that categorize the effects of known reproductive toxicants. These databases can be used to derive so-called read across information that allows extrapolation from a known toxicant to other related untested molecules. This can be complemented with other in silico, or in vitro test information on these compounds. We expanded databases that contain information relevant to reproductive toxicity in the RepDose (repeated dose) [15] and FeDTex (fertility and developmental toxicity) databases [16]. First, reproductive toxicity studies were identified from peer reviewed publications. All available studies on reproductive toxicity were selected for data entry. In particular, the amount of chemicals in FeDTex were more than doubled in the course of ChemScreen, mounting up to over 300. The expanded databases were used to identify redundant data coming from animal studies and thus possibilities to reduce testing. Evaluating the responsiveness of the different generations, a comparison of the dose levels at the No-Observed-Effect Level (NOEL) was performed. These data suggest a tendency of F0 and F1 being mostly equally sensitive in one- and multi-generational studies, whereas F2 seems to be less indicative for NOEL derivation [16]. This confirms earlier work on 2-generation study databases by Piersma et al. [17].

A significant overlap with RepDose was created to allow comparison of in vivo data with the same chemical and evaluate possible predictivity of repeated dose toxicity for reproductive toxicity. Target organs and effects observed within repeated dose toxicity were explored for any relationship to effects occurring in reproductive toxicity studies. Whether and how minimal effect concentrations of repeated dose toxicity can be extrapolated to reproductive toxicity appeared to depend on the chemical class of the compounds studied. For example, none of the repeated dose studies on chlorinated phenols (including phenol as parental substance) provided an indication for reproductive toxic effects., The major effects observed after repeated exposure were body weight loss related to reduced availability of energy and increased catabolism as well as changes 
in liver weight and pathology. The reproductive toxicity observed might also be related to insufficient energy supply due to uncoupled oxidative phosphorylation resulting in growth retardation or even increased prenatal mortality. The mechanism of toxicity is thus likely to be the same in repeated dose as well as reproductive toxicity but without mechanistic knowledge it is difficult to predict such effects based of repeated dose toxicity observations only. For alcoholic solvents, phthalates and glycol ethers comparable conclusions have been drawn [Batke et al., unpublished results].

Using the structural data of the PRS (see Section 1) a reproductive toxicity screen was carried out as well, however only covering a small part of reproductive toxicity (teratogenic effects by a limited number of mechanisms). The limited amount of compounds available for training and validation in combination with many different mechanisms and structural alerts hamper development of generally applicable QSAR models in this field [18].

Several tools were developed that can assist to predicting environmental reproductive toxicity of chemicals on a mechanistic basis aiming at more straightforward data interpretation [Dietrich et al., unpublished results]. By focusing on molecular mechanisms conserved in various species (e.g. various receptor-mediated processes) possibilities were explored to extrapolate molecular screening data to effects in aquatic organisms (particularly fish) and thus for ecotoxicological effects. For this, extrapolation methods have been designed to establish if there are conserved molecular mechanisms in aquatic organisms responsive to the action of different chemicals. As one of the first steps an expansion and restructuring of the large EDUKON ecotoxicology database [19] has been carried out as well as an inventory of possible chemobiological interactions that can be related to distinct molecular mechanisms. All relevant technical prerequisites to screen and to use the EDUKON DB as intended within the project description were fulfilled, and the integration of approximately 1400 new data sets of peer-reviewed studies into the DB was accomplished in accordance with the intention to integrate the newest scientific findings into the DB and to set it to the current state of science. The heterogeneity of the data involving many species and effects together with the complexities of reproductive toxicity, however, hampered the generation of firm conclusions on the applicability of mechanistic insights in environmental risk assessment [Dietrich et al., unpublished results].

\subsection{Establish sensitive parameters and a medium throughput in vitro assay panel}

The aim of this part of the project was the design of a reproductive toxicity screen, consisting of a number of in vitro assays that are representative of those parameters in reproductive toxicity that are crucial for reproductive hazard assessment. One of the starting points was a rather complex battery of tests originally developed in the FP6 ReProTect project using primary material and cells from various sources (e.g. rat embryos, oocytes, spermatocytes, etc) [7]. The first approach in simplification of the testing battery was directed towards inclusion of integrative medium throughput assays that can be run in a routine laboratory environment, avoiding assays with differentiated (primary) tissues as present in the ReProTect battery. This can then be combined with more high throughput assays (see next section) thereby enhancing throughput and further simplifying test procedures [20]. The next step would be to validate this battery and select the most promising assays needed to give proper predictions of reproductive toxicity of chemicals. Our approach in that sense is an intermediate between this ReProTect approach and that of ToxCast, which initially focused on a much larger variety of less selective high throughput screening (HTS) assays [21]. We expected that a smaller battery would reduce noise and allow more straightforward data interpretation. Validation steps subsequently can lead to identification of missing endpoints, and through inclusion of additional tests this battery can be further improved with time.

At the start of the project we envisioned that one way to simplify testing schemes is the approach of identifying critical endpoints for reproductive toxicity using literature and database searches, and focusing on development of tests for these endpoints. So far most endpoints identified were quite generic and non-specific in nature, which may mean that screening panels should also include quite simple, relatively non-specific tests (cytotoxicity) in addition to more mechanistic assays. We have therefore included separate cytotoxicity endpoints in all our screening assays and have extended the number of medium- and high throughput mechanistic screening assays.

First of all, progress was obtained with the optimization and amendment to higher throughput of tests that aimed to be included in the final battery, namely a genomics-based improved embryonic stem cell test (EST), the zebrafish embryotoxicity assay (ZET) and steroidogenesis assays, in particular four CYP17/19 assays. In the embryonic stem cell test, transcriptomics readouts were employed to improve the mechanistic readout of the system and improve its predictive value. The CYP17/19 assays were being optimized and microsomes versus whole cell assays were compared for their predictive value [22]. The embryonic stem cell assay was applied to the category of phthalates and predictivity within this class of compounds on a comparative basis with existing transcriptomics patterns of other phthalates was shown [23,24]. All above-mentioned tests were included in a first, relatively low throughput version of the ChemScreen battery [25].

\subsection{Establish a high throughput mechanistic pathway screen for reproductive toxicants}

A major focus was on the establishment of mechanistic tests and adapting them to high throughput screening methods predictive of reproductive toxic potential, based on insight in molecular mechanisms that are relevant to reproductive toxicity. These assays comprise a panel of highly specific human CALUX ${ }^{\circledR}$ reporter gene assays, which includes a selection out of about 50 different assays. This panel is based on the human U2-OS cell line which are modified to express reporter genes for separate pathways $[10,11]$. The high selectivity is reached through the use of reporter genes with synthetic promoters consisting of minimal promoter elements coupled to multimerised, highly selective response elements driving the expression of the reporter gene luciferase. When relevant to the pathway of interest, specific receptors are introduced to activate the cognate reporter gene. The panel of selective assays was designed to create very low levels of false-positives. The panel contains a single reporter gene assay per pathway. This is different in the much larger ToxCast assay panel that typically includes multiple assays per endpoint [26].

A systematic approach has been taken developing the screening panel using very sensitive and selective reporter genes, covering a wide range of receptors and signaling pathways that potentially are involved in reproductive toxicity [20]. The approach taken aimed to provide several advantages on top of a low rate of false positivity. First, by providing a mechanistic basis regulatory acceptance should be facilitated. Second, this mechanistic basis will provide input for decisions on further test requirements and risk assessment. Third, cost, speed, robustness, and quantification are advantageous in these types of assays. Finally, this approach facilitates predictions for both human- and ecotoxicological properties of chemicals, when different prediction models are used. One approach taken to develop additional reporter gene assays for ChemScreen is to use central intracellular pathways that are involved in regulating transcription in a mammalian cell. These 
pathways include besides nuclear hormone receptors also reporter gene assays for quite generic responses to toxicants, including cytotoxicity, apoptosis and genotoxicity. These responses can also be relevant in reproductive toxicity, however. A control cell line, called cytotox CALUX has been generated which constitutively expresses the luciferase gene [27]. To validate this approach of using limited sets of selective assays it was shown that a single selective and extensively validated CALUX assay (ERalpha CALUX) gave comparable results as a battery of ToxCast tests measuring estrogen receptor pathway-mediated endpoints [Van der Burg et al., unpublished results].

In addition to these highly selective CALUX HTS reporter gene assays, other assays have been established in more complex, murine embryonic stem (ES) cells by introducing reporter systems for signaling pathways controlling key differentiation pathways in embryonic development (so-called ReProGlo assays) [28]. Despite several attempts to introduce reporter genes other than that measuring wnt-pathway activation, as used in the original ReProGlo assay, none of them succeeded [Uibel et al., unpublished results].

All CALUX and ReProGlo assays have now been automated in 384 and 96 wells format, respectively, and a series of reference compounds has been used to deselect less informative assays and identify missing endpoints. Data storage and analysis has been set up and more than 250 compounds have been screened in the CALUX panel and a subset in the ReProGlo assay. Because of the need of a practical tool to store and process the large amount of high throughput data this part of the tool was generated. It provides the features required to store, manage and quickly analyze assay data generated in high HTS bioassays allowing access to primary data. This tool was successfully constructed and installed and all CALUX HTS screening data have been uploaded. Finally, the CALUX HTS panel endogenously expresses little metabolic activity but can be run with and without S9 metabolic fractions [27].

Our approach from the beginning onwards focused on inclusion of a limited number of tests with relevant modes of action. This is different from the ToxCast test panel that includes a wider range of mechanisms with multiple tests per mechanistic endpoint [21]. Results suggest that it may not be needed to include a range of assays for each endpoint to make good predictions of in vivo activity of chemicals, as was shown for the estrogen receptor reporter gene assays [20]. This is consistent with the earlier notion that transactivation of ERs in one assay, ER subtype or species can be extrapolated to other species or subtypes of ERs for the purpose of chemical screening in the current practice of risk assessment relying on relatively uninformative animal data [29]. Also in a panel the assays performed very well predicting reproductive toxicity in more general terms [25] which again suggests that a relatively simple screening panel can suffice for this purpose.

\subsection{Establish methods to predict in vivo reprotoxicity}

As a next step in our testing strategy we have been establishing integrative methods to predict in vivo reproductive toxicity concentrations using in vitro benchmark (threshold) concentrations as a starting point, also referred to as reverse dosimetry [30]. Prediction of the correct in vivo dose level at which adverse effects can be expected is one of the key issues. High throughput pharmacokinetic models have been set up describing the bioavailability via relevant routes (oral, dermal, inhalation) and the pharmacokinetics of the most relevant reference chemical classes. A rapid PBPK modeling strategy has been developed to translate in vitro concentrations to in vivo maternal dose levels ([31]; Fig. 3). Instead of building custom models for each chemical (class) as planned, it was chosen to develop one generic PBPK model framework describing all relevant compartments, physiological processes and exposure routes. The generic model with its (chemical-independent) anatomical and physiological parameter values in principle applies to all chemicals. The pharmacokinetics of specific compounds has been addressed by providing chemical-specific parameters (relating to permeability, partitioning and clearance) that can be obtained by either in silico predictions or routine in vitro measurements, as input to the generic model. To this end, an algorithm has been implemented to predict the binding and partitioning behavior of compounds in water, protein and lipid phases of blood (plasma and red blood cells) and tissues (interstitial fluid and cells). This algorithm takes as input readily obtainable chemical properties. It can also be used to predict binding and partitioning behavior in culture medium if its $\mathrm{pH}$ and protein and lipid fractions are known, and thus to correct for differences in unbound concentrations between in vitro and in vivo. This generic framework is more compatible with the rapid screening character of the ChemScreen tool as a whole, and allows pharmacokinetic prediction of a much larger number of compounds in a relatively short time [31].

In addition, to assess dermal exposure routes a specialized methodology for in vitro-dermal in vivo extrapolation was developed [32]. The method enables the calculation of an external exposure dose leading to systemic toxicity by combining in vitro experimental data relevant to the toxicant affected pathways with in silico predictions of skin permeation and whole-body ADME. The method is in line with the current development of mechanistic methods used to predict in vivo toxicity from sets of reference data, which may combine data from in vivo experiments, in vitro cell-based assays and/or computational results. Due to incomplete understanding of the mechanisms of toxicity and data limitations, determination of the representative concentration in vitro will most likely need to rely on a statistical approach of choosing the lowest value of EC50 distribution. The use of the internal systemic concentration external to estimate a safe dose has been compared to an approach based on in vivo dose (LOEL). Results suggest that LOELs may be too conservative for use in prediction of toxicity and risk assessment [32].

\subsection{Validation}

Acceptance by regulatory agencies is a major bottleneck for application of any alternative testing strategy. Therefore, during the project ample attention has been paid to validation and regulatory acceptance of the test methods developed in ChemScreen. This has been done in the context of the ring trails, but also formal validations using traditional methods have been undertaken. Close contacts have been established with relevant ECVAM and OECD working groups, scientists and regulators. In selecting tests to be included in the battery, methods were preferred that were either validated or in the process of being validated, including the EST test [33], the zebrafish embryo test [34], and reporter gene assays for estrogens [35,36] and androgens [37]. The ERalpha- and AR CALUX assay already were prevalidated in the context of the ReProTect FP6 program, and subsequently have been this issue to EURL-ECVAM and OECD to allow formal validation, and regulatory acceptance $[35,36,38,39]$. Since our battery of tests of reporter gene assays is very comparable to these latter tests, the validated tests may be used as "validation anchors" in a screening battery, i.e. giving confidence in the other battery constituents. It would also be possible to design more simple and rapid validation procedures for these additional battery constituents since the test procedures of all these tests essentially are the same.

An important element in the project from the beginning onwards was the execution of dedicated ring trails (also referred to as feasibility studies) to test and improve the test battery as a whole. Within the budgetary constraints, we chose to go for relatively small dedicated trails rather than an unbiased large screening with many assays and many chemicals. The first ring trail using the 


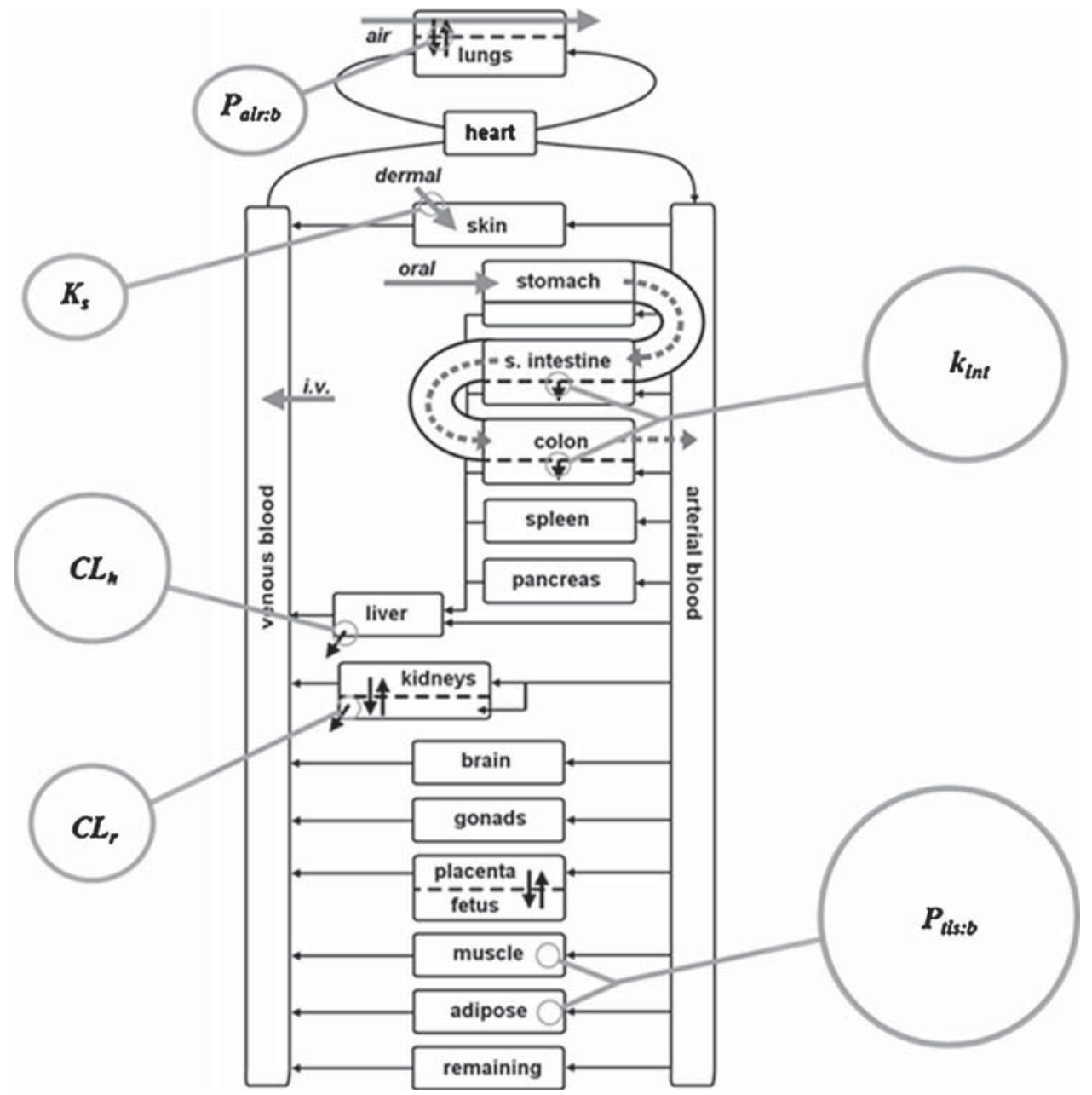

Fig. 3. Schematic representation of key elements of the high throughput PBPK model. For details see text and Bosgra et al., 2015.

complete preselected test battery showed that testing a limited list of compounds for which sufficient in vivo information is available, can be a valuable step in the identification of key elements in a battery as well as missing ones [25]. Correct predictions were made for 11 out of 12 chemicals while chemical that was missed could be explained by the lack of relevant mechanism of action being present in the testing battery. Interestingly, the predictions of the high throughput CALUX panel alone, when combined with PBPK modeling was exactly the same ([25]; Table 1)

QSAR and related chemoinformatics tools alone clearly are insufficient for reliable predictions of reproductive toxicity of chemicals. Similarly, biological methods in vitro screening methods have their limitations. When combined, predictions can greatly improve [40]. Therefore, we assessed further options to combine structural properties in combination with in vitro data to identify substances with reproductive toxic properties [41]. By using the OECD Toolbox [42] and expert judgment we identified 89 chemical categories. In addition 275 chemicals were identified with any effect on the reproductive system in males and female animals. We combined these results with a large data set on estrogen and androgen receptor activity using CALUX assays to determine if these hormonal effects are related to the reproductive aberrations. The in vivo and in vitro effects were found not to be confined to distinct chemical categories, showing the limitations of activity predictions on chemical structure only. While the chemical classes were not predictive for an in vivo effect, a clear correlation was seen between the in vivo reproductive aberrations and activity in the reporter gene assay on estrogenic activity [41].
A further application that was explored using a feasibility study was the possibility of read across using the ChemScreen battery: when an untested query chemical is structurally similar to a reproductive toxicant, the battery could be used to confirm these reproductive properties, and to avoid any further in vivo studies. Ideally, this battery should then also be capable to correctly distinct reproductive toxicants from non-reproductive toxicants, even when both have a clear structural similarity. This study showed that the battery was able to make this distinction using three different chemical classes as an example. This provides important opportunities for applications under REACH where read-across procedures are being considered as an important element of integrated testing to avoid or reduce animal experimentation [43]. Here the weight of evidence of available repeated dose toxicity data for both source and query chemical, their structural similarity, as well as their battery results could be used to justify waiving an in vivo study [42]. Alternatively or additionally, linkage to regulatory application may use combination of in vitro test results with existing repeated dose study data potential reproductive toxicants could be identified ahead of in vivo studies, allowing prioritizations of animal testing [41].

\subsection{Mechanistic validation and linkage to adverse outcome pathways}

Because of the strong interest in molecular screening approaches as developed in ChemScreen and ToxCast, currently there are significant activities ongoing to improve linkage of 
Table 1

Results from the first ChemScreen ring trail (adapted from Piersma et al. [25]).

\begin{tabular}{|c|c|c|c|c|c|c|c|c|c|}
\hline compound & $\begin{array}{l}\text { Toxicity } \\
\text { in vivo }\end{array}$ & $\overline{E S T}$ & $\overline{\text { ZET }}$ & ReProGlo & Сур17 & Сур19 & CALUX & $\begin{array}{l}\text { CALUX } \\
\text { PBPK }\end{array}$ & $\begin{array}{l}\text { Entire } \\
\text { battery }\end{array}$ \\
\hline Cyclosporin A & & & & & & & & & \\
\hline $\begin{array}{l}\text { Monoethylhexyl } \\
\text { phthalate }\end{array}$ & & & & & & & & & \\
\hline $\begin{array}{l}\text { Sodium } \\
\text { valproate }\end{array}$ & & & & & & & & & \\
\hline D-mannitol & & & & & & & & & \\
\hline Flusilazole & & & & & & & & & \\
\hline $\begin{array}{l}\text { Glufosinate } \\
\text { ammonium }\end{array}$ & & & & & & & & & \\
\hline $\begin{array}{l}\text { Methoxy acetic } \\
\text { acid }\end{array}$ & & & & & & & & & \\
\hline Retinoic acid & & & & & & & & & \\
\hline $\begin{array}{l}\text { Dioctyltin } \\
\text { chloride }\end{array}$ & & & & & & & & & \\
\hline Endosulfan & & & & & & & & & \\
\hline Diethylstilbestrol & & & & & & & & & \\
\hline $\begin{array}{l}\text { Methylmercury } \\
\text { chloride }\end{array}$ & & & & & & & & & \\
\hline
\end{tabular}

Gray: positive result; white: negative result; EST: embryonic stem cell test; ZET: zebrafish embryonic test; CALUX: CALUX HTS panel of reporter gene assays; battery: combined predictions of all tests.

mechanistic in vitro tools to results in animal experiments, e.g. through the design of so-called Adverse Outcome Pathways [44]. ChemScreen's scientists have embarked on these new and important activities thought to be essential missing links in application of molecular screening tools. We have been actively engaged in promoting regulatory acceptance of the tools developed, also using alternative validation methods that provide linkage to the mechanism of action of chemicals leading to adversity.

To this end, in a next feasibility study sets of chemicals were selected that can induce either sex organ (SO) deformities or neural tube deformities (NTD). On the one hand these were used to study the different adverse outcome pathways involved in neural tube defects [45], while on the other hand these sets of chemicals were used to further validate and specify the application domains of the different elements in the high throughput CALUX test battery [20]. Analysis of the results of the CALUX high throughput panel shows that it is very suitable to identify not only endocrine active compounds, but also can predict chemically induced SO deformations with over $80 \%$ accuracy [20]. This is consistent with the study of Lewin et al. [41], showing a clear correlation between the in vivo reproductive aberrations and activity in the reporter gene assay on estrogenic activity.

When using molecular screening tools it should be noted that coupling these results 1:1 to apical endpoints in experimental animals cannot be readily done. In the first place, there may be multiple mechanisms leading to the same endpoint, while the same mechanism may be involved in different endpoint toxicities when time and tissue distribution is different. In addition, a single test only gives part of the puzzle and only batteries such as the ChemScreen battery can be effective in replacing animal studies. The validation of such a battery is a challenge, in particular in the absence of gold standard, caused by relatively poor predictivity of animal data for humans [6]. An interesting approach in this light was to cluster apical reproductive toxic effects of chemicals when comparing this with data of molecular screening assays, resulting in a strong correlation of relevant CALUX assays with those clustered apical effects $[20,41]$. This very likely will challenge the design of very simple linear AOPs and suggest that network analysis and/or clustered AOP elements may be needed to link mechanistic tests to apical endpoints in an efficient manner.

In this context it is interesting to note that the ReProTect testing battery was able to provide predictions on gender-specific effects of the chemicals, and sometimes even the target organ [7]. This could be done because of inclusion of tests based on primary material of male and female reproductive organs. No attempts were made to predict gender-specific effects with the ChemScreen battery, but it is likely to be more difficult based on the apical tests included and the mechanistic tests because of their limited gender specificity. Even the results of CALUX assays that assess sex hormone receptor activation where found to be relevant for predicting reproductive effects in both male and female rodents [41], which is consistent with expression and function of AR and ER in both sexes. Therefore, although mechanistic tests can be highly predictive for in vivo toxicity, exact prediction of the apical endpoints that will be affected in animals will in general not be straight forward. The same problems, however, occur in extrapolating between animal species, life stages and organ toxicities. Mechanistic tests do have the opportunity to more directly link to human risk assessment, however. This can lead to better human risk assessment. For instance, estrogen signaling is complex and aberrant activation is associated with several human diseases properties not tested in standard animal tests [46,47]. In the future more mechanism-based risk assessment using tests providing more direct mechanistic information on human tissue-specific receptor 
activity and associated disorders may become an interesting opportunity.

\subsection{Application of the ChemScreen toolbox}

An important aim of the European framework programs is dissemination of results and their application, preferably leading to new business opportunities of small and medium enterprises. To reach this we worked on coupling different tools and databases in order to design integrated testing strategies that can be used in chemical risk assessment. The following modules were defined:

\subsubsection{Module 1. In silico prescreening and data collection module}

This module includes in silico prescreening (QSAR) methods to categorize chemicals into major classes of toxicity prioritized in REACH. Predictions for carcinogenicity, mutagenicity are applied, which in case of positivity will drive risk assessment in this direction avoiding reproductive toxicity testing according to $\mathrm{REACH}$ guidelines. It also uses established databases and literature to find any testing results of the chemical itself and use chemoinformatics to find structural analogues with reproductive toxicity data to be used as source chemicals in a read across approach.

\subsubsection{Module 2. In vitro screening module}

The test battery established within ChemScreen can provide confirmation of already predicted reproductive toxicity from in module 1 , or give a first alert of potential reproductive toxicity in the absence of predictions from these in silico methods. This screening model already gives reliable predictions for strong reproductive toxicants. Clearly, this promising screening module can be further improved when more mechanisms of reproductive toxicity are elucidated, allowing a greater reliance on the high throughput assays at the expense of the lower throughput ones.

\subsubsection{Module 3. Kinetic module}

The established PBPK tools link in vitro and in vivo plasma concentrations, and extrapolate these to associated oral, dermal or potentially inhalation doses. These are to be applied for validating and interpreting module 2 results, i.e. to classify chemicals, prioritize chemicals to be tested, and for helping to design limited in vivo studies for selected chemicals in case considered necessary.

\subsubsection{Module 4. Data integration and risk assessment}

All elements of the ChemScreen integrated testing strategy have been set up and are in an advanced state of being coupled and integrated. The efficacy of this integration in terms of making decisions on the need of subsequent in vivo animal tests, heavily depends on the purpose of the investigation, e.g. whether it is for safe design of new chemicals or for registration of existing chemicals. For instance, in the context of the REACH testing program read across can be a viable option, e.g. to reduce the reproductive toxicity testing requirement for chemicals (i.e. OECD 421, or 422) produced at more than 10 tons. The costs of this in vitro testing is around 10 times lower than the in vivo testing, which in its cheapest version costs around 55,000 Euro [48]. Expert judgment still is needed in different situations to interpret the outcomes, e.g. to verify the applicability domains of the models used, both with regard to structural as well as biological characteristics. Currently, more case examples are being run to further validate these integrated approaches.

Our test battery and integrated testing concept will be disseminated further, and training and support facilities are offered using the world-wide expertise available to disseminate bioassays. The coordinator at BioDetection Systems is the contact person for these activities (contact details; see www.chemscreen.eu).

\section{Conclusions and future directions}

The combined efforts of the ChemScreen consortium have led to a modular integrated assessment strategy which includes methods for in silico prescreening, in vitro screening, methods to extrapolate in vitro results to in vivo predictions, and integrative ones to combine these data with preexisting knowledge on the test chemical or related chemicals. This work confirms and extends the earlier finding of the FP6 ReProTect project [7] that a relatively simple battery of tests can be used successfully to predict reproductive toxicity of chemicalssee Table 1 . Significant advances have been made particularly in the further simplification of the test battery, while still being able to identify reproductive toxic chemicals efficiently, either in isolation [25], or in a grouping context $[41,43]$. We found that even with a relatively small number of tests, either apical tests combined with mechanistic tests or an relatively small number of mechanistic tests predictivities ranging from 74 to $94 \%$ can be reached $[20,25]$, which is comparable to that obtained with much larger ToxCast screening panels [21], the ReProTect battery [7], or the zebrafish ELS tests [49], a human embryonic stem cell test [50]. This is remarkable, since the concordance between different test species, like rabbit and rat has been estimated to be not more than $60 \%$ [6]. It seems that these validation results may be biased to some extent, e.g. because relatively strongly acting reproductive toxic chemicals are often used in the validation studies. Therefore, to create more confidence we have also developed and applied other means of validating tests using mechanistic knowledge on the tests and using selected chemicals to test whether the predicted adverse outcomes match with the mechanisms included in the battery [20]. More detailed classification schemes of chemicals, linked to both chemical and biological properties as developed by $\mathrm{Wu}$ et al. [40] may be very helpful in further validation and improvement of the test battery, through identification of chemical classes or mechanisms missed by the battery.

Although the ChemScreen battery showed a very good performance identifying strong reproductive toxicants, it is uncertain how it will perform with chemicals that have a less explicit activity profile. This is relevant, since the majority of industrial chemicals are likely to have relatively non-specific modes of action [51]. There is no reason to believe that these chemicals will be missed by the battery, since relevant pathways seem available. Also part of the reproductive toxic chemicals used in our first feasibility study do not act through specific mechanisms, and nevertheless are picked up through relatively non-specific cytotoxicity endpoints in our test battery [25].

Clearly not all pathways of reproductive toxicity are known at present, and the panel of mechanistic assays may therefore be missing compounds acting via less common mechanisms of action. On the other hand, it seems also likely that chemicals often address multiple pathways of toxicity, thereby reducing the need to have all possible mechanism of action included in a final battery. This is supported by the very good performance of our battery, even when compared with much larger batteries [21] or more integrative lower throughput batteries and tests [7,49,50]. At present the current combination of apical and HTS tests seems a pragmatic and effective choice that can be further improved when more mechanisms of reproductive toxicity become known. For regulatory acceptance, it may also be helpful to include test data of these more apical tests since initially they may give additional confidence in the test results and assist with classification and labeling, particularly in the absence of animal data. It should be noted that the majority of REACH chemicals are expected to be relatively inert and not to be reproductive toxic. Because of this, for regulatory application a wide coverage of possible mechanisms of action of toxicants is needed, but without giving many false-positives that may lead to additional testing requirement. Our battery of very selective 
mechanistic tests in combination with apical tests and available animal test data seems in this respect an effective testing strategy. With increased experience and confidence in in vitro testing the weight on in vitro testing and extrapolation to human rather than animal data will become increasingly important.

\section{Conflict of interest}

The authors declare that there are no conflicts of interest.

\section{Acknowledgments}

The work described in this manuscript could not have been exerted without the efforts of all additional ChemScreen participants not specifically mentioned. We would particularly like to sincerely thank our advisory board members Drs David Dix, Robert Chapin, Maria Bondesson, Mark Cronin, and Neil Carmichael for their support and valuable contribution to the success of the ChemScreen project. We are also very grateful for the contribution of Drs Burkhard Flick, Thomas Sobanski and Sander van der Linden to the final symposium and a panel discussion that provided elements of this review. This work was carried out with financial support from the Commission of the European Communities, the collaborative project ChemScreen (GA244236).

\section{References}

[1] European Commission. White paper "strategy for a future chemicals policy". COM 2001;88:2001.

[2] European Commission (EC). Regulation (EC)No1907/2006 of the European Parliament and of the Council of 18 December 2006 concerning the Registration, Evaluation, Authorization and Restriction of Chemicals (REACH), establishing a European Chemicals Agency, amending Directive No 1488/94 as well as Counci Directive 76/769/EEC and Commission Directives 91/155/EEC, 93/105/EC and 2000/21/EC. Off J Eur Union L 2007 2007:136:3-280.

[3] Hartung T, Luechtefeld T, Maertens A, Kleensang A. Food for thought ... integrated testing strategies for safety assessments. ALTEX 2013;30(1):3-18.

[4] Van der Jagt K, Munn S, Torslov J, de Bruijn J. Alternative approaches can reduce the use of test animals under REACH European Commission Report EUR 21405; 2004.

[5] Costanza R, Hartung T. Re-evaluation of animal numbers and costs for in vivo tests to accomplish REACH legislation requirements for chemicals-a report by the transatlantic think tank for toxicology. ALTEX 2009;26:187-208.

[6] Hurtt ME, Cappon GD, Browning A. Proposal for a tiered approach to developmental toxicity testing for veterinary pharmaceutical products for food-producing animals. Food Chem Toxicol 2003;41(5):611-9.

[7] Schenk B, Weimer M, Bremer S, van der Burg B, Cortvrindt R, Freyberger A, et al. The ReProTect Feasibility Study, a novel comprehensive in vitro approach to detect reproductive toxicants. Reprod Toxicol 2010;30:200-18.

[8] National Research Council (USA). Scientific frontiers in developmental toxicology and risk assessment. Washington, DC: NAS Press; 2000.

[9] National Research Council (USA). Toxicity testing in the twenty-first century: a vision and a strategy Committee on Toxicity and Assessment of Environmental Agents. Washington, DC: National Research Council; 2007.

[10] Sonneveld E, Jansen HJ, Riteco JAC, Brouwer A, Van der Burg B. Development of androgen- and estrogen-responsive bioassays, members of a panel of human cell line-based highly selective steroid responsive bioassays. Toxicol Sci 2005;83:136-48.

[11] Sonneveld E, Riteco JAC, Jansen HJ, Pieterse B, Brouwer A, Schoonen WG, et al. Comparison of in vitro and in vivo screening models for androgenic and estrogenic activities. Toxicol Sci 2006;89:173-87.
[12] Sonneveld E, Pieterse B, Schoonen W, Van der Burg B. Validation of in vitro screening models for progestagenic activities: inter-assay comparison and correlation with in vivo activity in rabbits. Toxicol In Vitro 2011;25:54554.

[13] Van der Burg B, Kroese ED, Piersma AH. Towards a pragmatic alternative testing strategy for the detection of reproductive toxicants. Reprod Toxicol 2011;31(May (4)):558-61

[14] Wedebye EB, Dybdahl M, Nikolov NG, Jónsdóttir SO, Niemelä JR. QSAR pre-screen of 70,983 substances for genotoxic carcinogenicity, germ cell mutagenicity, developmental toxicity and endocrine activity in the ChemScreen project. Reprod Toxicol 2015;55:64-72.

[15] Bitsch A, Jacobi S, Melber C, Wahnschaffe U, Simetska N, Mangelsdorf I. REPDOSE: a database on repeated dose toxicity studies of commercial chemicals-a multifunctional tool. Regul Toxicol Pharmacol 2006;46(3):202-10.

[16] Schulz F, Batke M, Mangelsdorf I, Pohlenz-Michel C, Simetska N, Lewin G. Sensitivity of different generations and developmental stages in studies on reproductive toxicity. Toxicol Lett 2014;226(Apr (2)):245-55.

[17] Piersma AH, Rorije E, Beekhuijzen ME, Cooper R, Dix DJ, Heinrich-Hirsch B, et al. Combined retrospective analysis of 498 rat multi-generation reproductive toxicity studies: on the impact of parameters related to F1 mating and F2 offspring. Reprod Toxicol 2011;31(May (4)):392-401.

[18] Novic M, Vracko M. QSAR models for reproductive toxicity and endocrine disruption activity. Molecules 2010;15(3):1987-99.

[19] 〈http://edukon.biologie.uni-konstanz.de/edukon/index.php〉.

[20] Van der Burg B, Pieterse B, Buist H, Lewin G, Van der Linden SC, Man HY, et al. A high throughput screening system for predicting chemically-induced endocrine disruption and sex organ deformities. Reprod Toxicol 2015;55:95-103.

[21] Sipes NS, Martin MT, Reif DM, Kleinstreuer NC, Judson RS, Singh AV, et al. Predictive models of prenatal developmental toxicity from ToxCast high throughput screening data. Toxicol Sci 2011;124(Nov (1)):109-27.

[22] Roelofs MJ, Piersma AH, van den Berg M, van Duursen MB. The relevance of chemical interactions with CYP17 enzyme activity: assessment using a novel in vitro assay. Toxicol Appl Pharmacol 2013;268(May (3)):309-17.

[23] Schulpen SH, Robinson JF, Pennings JL, van Dartel DA, Piersma AH. Dose response analysis of monophthalates in the murine embryonic stem cell test assessed by cardiomyocyte differentiation and gene expression. Reprod Toxicol 2013:35(Jan):81-8.

[24] Schulpen SH, Pennings JL, Tonk EC, Piersma AH. A statistical approach towards the derivation of predictive gene sets for potency ranking of chemicals in the mouse embryonic stem cell test. Toxicol Lett 2014;225(3):342-9.

25] Piersma AH, Schulpen SHW, Uibel F Van Vugt-Lussenburg B, Bosgra S, Hermsen $\mathrm{SAB}$, et al. Evaluation of an alternative in vitro test battery for detecting reproductive toxicants. Reprod Toxicol 2013;38:53-64.

[26] Dix DJ, Houck KA, Martin MT, Richard AM, Setzer RW, Kavlock RJ. The ToxCast program for prioritizing toxicity testing of environmental chemicals. Toxicol Sci 2007;95(1):5-12.

[27] Van der Linden SC, von Bergh AR, van Vugt-Lussenburg BM, Jonker LR, Teunis M, Krul CA, et al. Development of a panel of high-throughput reporter-gene assays to detect genotoxicity and oxidative stress. Mutat Res Genet Toxicol Environ Mutagen 2014;760:23-32.

[28] Uibel F, Mühleisen A, Köhle C, Weimer M, Stummann TC, Bremer S, et al. ReProGlo: a new stem cell-based reporter assay aimed to predict embryotoxic potential of drugs and chemicals. Reprod Toxicol 2010;30(1):103-12.

[29] Dang Z, Ru S, Wang W, Rorije E, Hakkert B, Vermeire T. Comparison of chemical-induced transcriptional activation of fish and human estrogen receptors: regulatory implications. Toxicol Lett 2011;201(Mar (2)):152-75.

[30] Wetmore BA. Quantitative in vitro-to-in vivo extrapolation in a high-throughput environment. Toxicology 2014, http://dx.doi.org/10.1016/ j.tox.2014.05.012, pii: S0300-483X(14)00114-0. [Epub ahead of print].

[31] Bosgra S, Westerhout J. Interpreting in vitro developmental toxicity test battery results: the consideration of toxicokinetics. Reprod Toxicol 2015;55:73-80.

[32] Dancik Y, Troutman JA, Jaworska J. Estimation of safe in vivo dose of dermally applied chemicals-illustration with four reproductive toxicants. Reprod Toxicol 2015;55:50-63.

[33] Seiler AE, Spielmann H. The validated embryonic stem cell test to predict embryotoxicity in vitro. Nat Protoc 2011;6(7):961-78

34] OECD. Test No. 236: Fish Embryo Acute Toxicity (FET) Test. OECD; 2013.

[35] OECD. Test guideline No.455. Stably transfected human estrogen receptor-transcriptional activation assay for detection of estrogenic agonist-activity of chemicals. Paris: Organisation for Economic Cooperation and Development; 2009.

[36] OECD. SPSF by the Netherlands: U2-OS cells Transcriptional ERalpha CALUX ${ }^{\circledR}$ assay for the detection of estrogenic and antiestrogenic chemicals for inclusion in TG455/TG457. OECD; 2013

[37] OECD. SPSF by the European Commission: performance-based test guideline on androgen receptor transactivation assays (AR CALUX). OECD; 2013.

[38] Van der Burg B, Winter R, Man H-y, Vangenechten C, Weimer M, Berckmans $P$, et al. Optimization and prevalidation of the in vitro AR CALUX method to test androgenic and antiandrogenic activity of compounds. Reprod Toxicol 2010;30:18-24.

[39] Van der Burg B, Winter R, Weimer M, Berckmans P, Suzuki G, Gijsbers L, et al. Optimization and prevalidation of the in vitro ER $\alpha$ CALUX method to test estrogenic and antiestrogenic activity of compounds. Reprod Toxicol 2010;30:73-80. 
[40] Wu S, Fisher J, Nacliff J, Laufersweiler M, Lester C, Daston G, et al, Framework for identifying chemicals with structural features associated with the potential to act as developmental or reproductive toxicants. Chem Res Toxicology 2013;26:1840-61.

[41] Lewin G, Escher SE, Van der Burg B, Simetska N, Mangelsdorf I. Structural features of endocrine active chemicals - A comparison of in vivo and in vitro data. Reprod Toxicol 2015;55:81-94.

[42] 〈http://www.oecd.org/chemicalsafety/risk-assessment/theoecdqsartoolbox. htm $\rangle$.

[43] Kroese DE, Bosgra S, Harrie E Buist, Van der Linden SC, Man HY, Piersm AH, et al. Evaluation of an alternative in vitro test battery for detecting reproductive toxicants in a grouping context. Reprod Toxicol 2015;55:11-9.

[44] Ankley GT, Bennett RS, Erickson RJ, Hoff DJ, Hornung MW, Johnson RD, et al. Adverse outcome pathways: a conceptual framework to support ecotoxicology research and risk assessment. Environ Toxicol Chem 2010;29(3):730-41.

[45] Tonk EC, Pennings JL, Piersma AH. An adverse outcome pathway framework for neural tube and axial defects mediated by modulation of retinoic acidhomeostasis. Reprod Toxicol 2014 Oct 16., http://dx.doi.org/10.1016/ j.reprotox.2014.10.008, pii: S0890-6238(14)00261-5 [Epub ahead of print] Review.
[46] Deroo BJ, Korach KS. Estrogen receptors and human disease. J Clin Invest 2006;116(Mar):561-70

[47] Heldring N, Pike A, Andersson S, Matthews J, Cheng G, Hartman J, et al. Estrogen receptors: how do they signal and what are their targets. Physiol Rev 2007;87(Jul):905-31.

[48] Rovida C, Hartung T. Re-evaluation of animal numbers and costs for in vivo tests to accomplish REACH legislation requirements for chemicals-a report by the transatlantic think tank for toxicology (t(4)). ALTEX 2009;26(3):187-208.

[49] Panzica-Kelly JM, Zhang CX, Augustine-Rauch K. Zebrafish embryo developmental toxicology assay. Methods Mol Biol 2012;889:25-50.

[50] Palmer JA, Smith AM, Egnash LA, Conard KR, West PR, Burrier RE, et al. Establishment and assessment of a new human embryonic stem cell-based biomarker assay for developmental toxicity screening. Birth Defects Res B: Dev Reprod Toxicol 2013;98(4):343-63.

[51] Thomas RS, Philbert MA, Auerbach SS, Wetmore BA, Devito MJ, Cote I, et al. Incorporating new technologies into toxicitytesting and risk assessment: moving from 21st century vision to a data-driven framework. Toxicol Sci 2013;136(1):4-18. 